\title{
A Novel Established Pyroptosis-participant Genes Signature for Predicting the Prognosis of Idiopathic Pulmonary Fibrosis
}

\section{Qian He}

Third Affiliated Hospital of Soochow University

\section{Yi Cao}

Changzhou Maternal and Child Health Care Hospital Hospital

\section{Yue Zhao}

Changzhou Maternal and Child Health Care Hospital Hospital

\section{Yi Shen}

Third Affiliated Hospital of Soochow University

\section{Xiong Xu}

Third Affiliated Hospital of Soochow University

Qiu-Di Zhang ( $\sim$ zhang.qiudi@163.com )

Third Affiliated Hospital of Soochow University

\section{Research Article}

Keywords: Idiopathic pulmonary fibrosis, Pyroptosis, Transcriptomics

Posted Date: December 21st, 2021

DOI: https://doi.org/10.21203/rs.3.rs-1129674/v1

License: (1) This work is licensed under a Creative Commons Attribution 4.0 International License. Read Full License 


\section{Abstract}

Idiopathic pulmonary fibrosis (IPF), caused by a strange reason and characterized by a bad clinical prognosis, is an advanced, long-time, and irreversible interstitial lung disease (ILD). In recent years, it has been confirmed that a pyroptosis is a phlogistic form of programmed cellular demise. At the same time, the expression of the pyroptosis-participant gene (PPG) in IPF and their pertinence behind prognosis remains indistinct. In this research, we identified 17 pyroptosis regulators that were distinguishingly expressed entre IPF and controls. The ground on these distinguishingly expressed genes (DEGs), entire IPF conditions could be uncoupled into two subtypes. The prognostic significance of respective PPG for surviving was assessed to find a polygenes signature doing with a Gene Expression Omnibus (GEO) pack (GSE28042). Via putting into use the least absolute shrinkage and selection operator (LASSO) Cox regression approach, a 6-gene signature was structured and ranged entire IPF sicks in GSE28042 into a shallow-threat or high-threat group. IPF sicks in the shallow-threat group revealed meaningfully upper survival chances than those in the high-threat group (pvalue < 0.001). Exploiting the mid threat-score from GSE28042, IPF sicks from another GEO cohort(GSE70866-GPL17077) were separated into two threat subgroups, and the shallow-threat set had augmented overall survival $(O S)$ time $(P=0.0018)$. United the clinical characteristics, the threat-score was a sole element for forecasting the overall survival of IPF sicks. Function enrichment analyses bespoke that modification of morphology or physiology of other organisms, killing of cellular of other organisms, and disruption of cellular of other organisms biological processes were increased in the high-threat group. GSEA(Gene Set Enrichment Analysis) showed that cancer- and autoimmune disease-participant "KEGG" gene sets were highly enriched in the high-threat phenotype. In general, PPGs play a crucial role in IPF and can be done to forecast the prognosis of IPFs, and our consequences suggest that the high-threat group of IPF may be linked with the response of other organisms and autoimmunity as well.

\section{Introduction}

Idiopathic pulmonary fibrosis (IPF), a chronic and ascensive lung disease of sealed nosetiology[1], is characterized by diffuse alveolar inflammation and alveolar configurational lesion [2]. The global yearly incidence of IPF is $0.2-93.7$ per 100,000 [3], and the mid survival time is only 2-3 years after diagnosis is confirmed [4]. Clinical biomarkers strictly indicating the advancement of IPF are small in number. In accordance with therapy, pharmaceuticals introduced by recent clinical guidelines - nintedanib and pirfenidone have an excellent treated influence on IPF in conclusion [5] but are not that available for latestage IPF. Accordingly, it is essential to clarify the etiopathogenesis of IPF and to detect feasible biomarkers for early-stage IPF to moreover the treated influence on IPF, and responsible novel prediction patterns are imminently needed to make directed therapies more possible.

Pyroptosis, likewise known as cellular phlogistic thanatosis, is a new way of programmed cellular destruction [6]. Pyroptotic cellular is featured by cellular inflation and lots of foam-like hernias. Under electron microscopy, pyroptotic cellular initially forms massive vesicles. Since these vesicles are arranged, holes are shaping in the cellula panniculus, which fractures and the contents shed [7]. The 
gasdermin family is the elementary performer of pyroptosis and involves gasdermin-A to gasdermin-E and PJVK [8]. Gasdermin family proteins are able to be trimed and polymerized, that consequence in disruption of the $\mathrm{N}$-tail-end and $\mathrm{C}$-tail-end chiasmatic configurational regions and liberation of alive $\mathrm{N}$ terminal territories; these territories bond cytomembrane greases, phosphatidylinositol, and cuorin and concentrate into the shallow holes in the cellula cytomembrane $[9,10]$. The gasdermin family albuminoid constitutes 10 to $20 \mathrm{~nm}$ shallow holes in the cellula panniculus, and cellula properties are lentamente unlocked via the cytomembrane shallow holes and produce expanded phlogistic responses. Cellular stage by stage triumph over, breeding 1-5 $\mu \mathrm{m}$ apoptotic vesicle-like hernias (burnt vesicles), and cellular step by step intumesce by the end the adtevak panniculus fractures, with characteristics such as nuclear compression and chromatin DNA destruction $[11,12]$. Pyroptosis was originally a pivotal theory for fighting infection, and many developmental studies advise that it likewise plays an essential mission in developing other refractory diseases. Records have shown that phlogistic vesicles, gas-sensitive proteins, and pro-phlogistic cytokines, critical pyroptosis components, are linked with IPF [13]. On the side, different apoptosis, as pyroptosis happens, varieties of hazard-linked signaling motes and cell factors are lively and unlocked, attended via a robust phlogistic reaction and revitalite of the immunizing scheme [14].

Given the existing findings, Lycorine (LYC) ameliorated bleomycin (BLM-) induced pulmonary fibrosis by damping NLRP3 phlogistic corpuscle activation and pyroptosis through targeting the pyrin domain of apoptosis-linked dust-like protein embracing a CARD [15]; whereas, detailed pyroptosis functions in IPF have been minor studied. Accordingly, we implemented scientific research to conclude the expression levels of PPGs inter IPF and controls, probe into the prognostic importance of these genes, and research the pertinence inter pyroptosis and the participant signaling pathway as well as the immune microenvironment.

\section{Consequences}

\section{Authentication of DEGs inter IPF and normals}

The 33 PPGs expression levels were contradistinguished in the GSE28042 dataset from 19 normal and 75 IPF organizations, and we identified 17 distinguishingly expressed genes $(D E G s)(P<0.05)$. Among them, four genes (SCAF11, PJVK, AIM2, CASP3) were downregulated, while 13 other genes (CASP4, CASP1, CASP9, NOD2, PRKACA, TIRAP, GSDMD, TNF, PYCARD, NLRP3, NLRC4, IL1B, and ELANE) were concentrated in the IPF group. The RNA levels of these genes are rendered as a heatmap in Figure $1 \mathrm{~A}$ (blue: shallow expression criterion; red: high expression criterion) and Figure 1B. To moreover probe into the mutual effect of these PPGs, we regulated a protein-protein interaction (PPI) analysis, and the consequences are given in Figure $1 \mathrm{C}$. The shallowest necessary reciprocity score for the PPI analysis was fixed at 0.9 (the supreme degree of confidence), and we resolved that NLRP1, CASP1, NLRP3, NLRC4, CASP8, CASP5, PYCARD, and AIM2 were center genes. In the midst of them, with the exception of NLRP1, CASP5, and CASP8, other genes were entire the DEGs inter control and IPFs (Figure 1D). The associations' network involved in all PPGs is rendered in Figure 1E (The thicker the line, the more significant the pertinence, and the more connected each node, the larger the node). 


\section{IPF category ground on the DEGs}

To probe into the joints inter the expression of the 17 pyroptosis-participant DEGs (PPDEGs) and IPF subtypes, we implemented an accordance clustering analysis with all 75 IPF sicks in the GSE28042 cohort. Via adding the variable clustering factor ( $k$ ) from 2 to 6 , we detected that since $k=2$, the intracommunity pertinence was, the supreme and the intra-community pertinence were shallow, reflecting that the 75 IPF sicks could be properly separated into two clusters ground on the 17 PPDEGs (Figure 2A, B, C). The gene expression profile and the clinical characteristics, incorporating the gender (male or female), age (>60 or $\leq 60$ years old), and living states (living or dead), are rendered in a heat map, whereas we detected there are few diversities in clinical characteristics inter the two clusters Figure 2D. The OS time was likewise contradistinguished inter the two clusters, whereas no apparent deviations were detected ( $P$ $=0.45$, Figure $2 \mathrm{E}$ ).

\section{The exploitation of a PPG pattern in the GSE28042 data set}

Amount of 75 IPF specimens were suitable with the suiting sicks who had whole survival data. Singlevariable Cox regression analysis was utilized for elementary filtration of the surviving-participant genes. The six genes (GSDMD, CASP8, CASP3, NLRP3, PYCARD, and PJVK) that met the conditions of $P<0.01$ were reserved for moreover analysis, and in the middle of them, five genes (GSDMD, CASP8, CASP3, NLRP3, and PYCARD) were linked with the increased threat with hazard ratios $>1$, but the else one genes (PJVK) were phylactic genes with hazard ratios $<1$ (Figure 3A). By executing the LASSO Cox regression analysis, a 6-gene risk scoring prediction model was found foundation the optimal $\lambda$ value (Figure 3B, C). The threat-score was computed as follows: threat-score $=(4.7739931 * \mathrm{NLRP} 3$ exp. $)+$ $(-11.1024476 *$ PYCARD exp. $)+(0.4391282 * P J V K$ exp. $)+(4.7534338 *$ GSDMD exp. $)+(2.4072683 *$ CASP3 exp. $)+\left(1.1296503^{\star}\right.$ CASP8 exp.). On the ground of the mid score calculated by the threat-score equations, 75 sicks were fairly separated into shallow-threat and high-threat subgroups (Figure 3D). The principal constituent analysis (PCA) revealed that sicks with diverse threats were divided into two clusters (Figure $3 \mathrm{E})$. Sicks in the high-threat group had additional mortality and a curter survival time than those in the shallow-threat group (Figure 3F, on the right flank of the dummy line). A remarkable diversity in OS time was spotted inter the shallow-threat and high-threat sets (pvalue $<0.01$, Figure $3 \mathrm{G}$ ). Receiver operating characteristic (ROC) analysis was implemented to assess the susceptivity and specificity of the prognostic pattern, and we detected that the area under the ROC curve (AUC) was 0.79 (Figure $3 \mathrm{H}$ ).

\section{Outer verification of the signature of threat}

Amount of 65 IPF sicks from a GEO dataset (GSE70866-GPL17077) were made use of as the verification dataset. Until moreover analysis, the gene expression profile was standardized by the "Scale" function. The ground on the mid-value threat-score in the GSE28042 cohort, 46 sicks in the GSE70866-GPL17077 cohort were divided into the shallow-threat group, whereas the other 18 sicks were divided into the highthreat group (Figure 4A). The PCA revealed the content distance inter the two subgroups (Figure 4B). sicks in the shallow-threat subgroup (Figure $4 \mathrm{C}$, on the left flank of the dummy line) were detected to have longer survival time and shallower demise proportions than those in the high-threat subgroup. On the 
side, the Kaplan-Meier analysis likewise bespoken a significant diversity in the survival proportion inter the shallow-threat and high-threat sets $(P=0.0018$, Figure 4D). ROC curve analysis of the GSE70866GPL17077 dataset revealed that our pattern possessed fine sibylline effectiveness (AUC =0.68) (Figure 4E).

\section{prognostic premonitory value of the threat pattern}

We employed single-variable and polynary Cox regression analyses to assess if the threat-score gotten from the PPG signature pattern could do duty for an autocephalous prognostic element. The singlevariable Cox regression analysis bespoke that the threat-score was a prognostic element forecasting bad survival in the GSE28042 and GSE70866-GPL17077 datasets (hazard ratio = 3.488, 95\% Cl: 1.64-7.421 and hazard ratio: 3.463, 95\% Cl: 1.508-7.827, Figure 5A, C). The polynary analysis likewise suggested that, after aligning for other farrago factors, the threat-score as a prognostic element (hazard ratio $=$ 3.7596, 95\% Cl: 1.6832-8.398 and hazard ratio: 3.396, 95\% Cl: 1.4807-7.789, Figure 5B, D) for sicks with IPF in both datasets. On the side, we created a heatmap of clinical features for the GSE28042 dataset (Figure 5E) and spotted that the living states were variously distributed inter the shallow-threat and highthreat subgroups (pvalue <0.05).

\section{Functional enrichment analyses ground on the threat pattern.}

To moreover probe into the diversities in the gene functions and pathways inter the subgroups divided by the threat pattern, we employed the "limma" R package to screen the differentially expressed genes by putting into use the criterion padj $<0.05$ and $|\log 2 \mathrm{FC}| \geq 1$. In sum, 43 differentially expressed genes inter the shallow-threat and high-threat groups in the GSE28042 dataset were recognized. Among them, 30 genes were up-reguline in the high-threat set, whereas the other 13 were reduced (the consequence is demonstrated in Table S2). GO enrichment analysis and KEGG pathway analysis were implemented ground on these differentially expressed genes. The consequences bespoke that the DEGs were primarily correlative with the modification of morphology or physiology of another organism, killing of cellular of another organism, and disruption of cellular of other organisms and other biological processes (padj<0.001) in the high-threat group (Figure 6A). The most enriched terms in cellular components were specific granule lumen, hemoglobin complex, specific granule, secretory granule lumen, and cytoplasmic vesicle lumen (Figure 6B). The most typical term in molecular functions were haptoglobin binding, oxygen carrier activity, oxygen binding, peroxidase activity, and molecular carrier activity(Figure 6C). In KEGG enrichment analysis, no statistically significant pathway was found (padj>0.05) (Figure 6D).

\section{Gene Set Enrichment Analysis (GSEA)}

GSEA was implemented to check into the concerning signaling pathways using the pyroptosis patternbased threat-score for classification. The consequences recommended that cancer- and autoimmune disease-participant "KEGG" gene sets, such as bladder cancer, systemic lupus erythematosus (Figure 7AB). 


\section{To compare the immune event inter subgroups.}

The ground on the functional enrichment analyses, we moreover contradistinguished the enrichment scores of 22 types of immune cellular inter the shallow-threat and high-threat groups in the GSE28042 and the GSE70866-GPL17077 cohorts by employing the CIBERSORT. In the GSE28042 cohort (Figure 8A, $B)$, the high-threat subgroup commonly had shallower levels of $T$ cellular regulatory Tregs than the shallow-threat subgroup, and that had high levels of monocytes, mast cellular resting. Whereas, when evaluating the immune conditions in the GSE70866-GPL17077 cohort, the high-threat subgroup commonly had higher levels of T cellular CD4 memory activated (Figure 9A, B). The consequences of the two cohorts were not consistent, perhaps suggesting that the level of immune infiltration is not an essential factor in the excellent prognosis of IPF sicks.

\section{Discussion}

This study, in the first place, researched the mRNA expression levels of 33 commonly known PPGs in IPF and controls and detected that more than half of them were distinguishingly expressed. In contrast, the two clusters manufactured by the accordance clustering analysis ground on the differentially expressed genes didn't significantly differ in clinical traits. To moreover assess the prognostic importance of these pyroptosis-participant adjusters, we found a 6-gene threat signature by way of Cox single-variable analysis and LASSO Cox regression analysis, which was then recognized to accomplish fine in an outer cohort. The functional researches have bespoken that the distinguishingly expressed genes inter the shallow-threat and high-threat groups were connected with the response of other organisms. The immune cellula infiltration in the shallow-threat and high-threat sets was contradistinguished, and we detected that the consequences of the two cohorts were not consistent.

Pyroptosis, an exquisite modality of programmed cellular demise, was found to play a two-tier character in IPF exploitation and curative mechanisms in latest years. For another, the suppression of IPF cellula pyroptosis might be a novel treated target [13]. In IPF, how PPGs mutual effect and if they are connected with the survival time of sicks keep up sealed. Our research created a signature featuring six PPGs (GSDMD, CASP8, CASP3, NLRP3, PYCARD, and PJVK) and found that it could forecast OS in IPF sicks.

PJVK is the mere insider of the gasdermin family that is short of the C-terminal domain, and it isn't distinct if this protein can produce cytomembrane punching and implement pyroptosis [16]. Because it is an insider of the gasdermin family and possesses a whole $\mathrm{N}$-terminal domain, we regarded it as a "feasible" PPGs. PJVK proved to be joint with absurdity, whereas its part in IPF has been little probed into [17]. We detected that PJVK was reduced in IPFs, and its shallow expression signed bad survival ratios, reflecting which it may act as an IPF suppresser gene in current research. Interestingly, PJVK was likewise underexpressed in the high-threat set in the GSE28042 queue.

Moreover, researches may be centered on if/how PJVK takes part in pyroptosis and IPF. GSDMD constitutes the precursor of the pore-forming protein: upon fragmentation, the released N-terminal onehalf (Gasdermin-D, N-terminal) bonds to cytocytomembranes and made pinholes, generating pyroptosis. 
In 2015, Shi J et al. [18] discovered that GSDMD promotes pyroptosis in answer to microbial infection and threat signals. Our study detected that GSDMD was up-regulated in IPF tissues, and its high expression signed bad alive rates, reflecting that it may act as an IPF stimulate gene in this study. CASP8 gaps and activates maneuvering gear caspases CASP3, CASP4, CASP6, CASP7, CASP9, and CASP10 [19]. CASP8 can institute pyroptosis by mediating disruption and excitation of gasdermin-D (GSDMD): GSDMD fragmentation promotes the liberation of the N-terminal one-half (Gasdermin-D, N-terminal) that bonds to cytomembranes and fosters stomates, generating pyroptosis [20]. Promoter protease induces apoptosis by mediating TNFRSF6/Fas-mediated and TNFRSF1A-induced cellula death effects. The cleavage and activation of caspases induce apoptosis [21]. CASP8 was up-regulated in sicks with decreased alive rates in our study. CASP3 appears as an unactivated proenzyme in its standard state. Still, in the light of activation, it turns out positive performers that gap tectonic and administrative proteins in the cytoplasm and tenuigenin of cellulases, thus managing cellular demise, and it is acknowledged as a sign of apoptosis [22]. In 2017, researchers [23] found out which GSDME was ripped, particularly by chemotherapeutic drug-active CASP3 to attract cytomembrane-osmolar GSDME-N debris, which placed a premium on pyroptosis.

The interesting thing is, in our research, CASP3 was as if an IPF-suppressing gene since it was downregulated in IPF tissues; whereas, it likewise conduced to shortening sick survival rates, and it was also gathered in the high-threat set. As the transducer module of the NLRP3 phlogistic corpuscle, NLRP3 plays an essential role in inherent phylactic power and phlegmonous. In answer to the pathogenic agent and miscellaneous lesion-linked signals, the phlogistic corpuscle converged complex whole initiates the constitution, composed of PYCARD, NLRP3, and CASP1 (and probably CASP4 and CASP5) [25]. Revitalization of the NLRP3 phlogistic corpuscle is likewise called for HMGB1 excretion [26], and phlogistic corpuscles can likewise cause pyroptosis. Under dormant circumstances, NLRP3 is autoinhibited. NLRP3 revitalize salts involve extracorpuscular ATP, corresponsive oxygen kinds, $\mathrm{K}+$ flux, the glutamate or cholesterin crystals, beta-amyloid fibers, surrounding perhaps industrial motes, nanoparticles, cellular solute dsRNA, etc. Nevertheless, it is indistinct what makes up the straight NLRP3 catalyst. Excitation Coram cytosolic dsRNA is mediated by DHX33 [27]. NLRP3 was up-regulated in sicks with decreased alive rates in our study. PYCARD, containing macrophage pyroptosis, is the supreme component of the ASC pyroptosome, which forms on the basis of potassium consumption and promptly recruits and activates caspase-1[28]. We detected that high PYCARD expression was linked with worse survival endings, given by its active regulation of pyroptosis.

In short, three genes (CASP8, CASP3, and NLRP3) in the premonitory pattern were certified to be pyroptosis merchandisers, and three genes (PYCARD, PJVK, GSDMD) were recognized as reasonable pyroptosis performers. Whereas these merchandisers and performers were not entirely linked with the bad IPF prognosis in our research. What way these genes mutually affect one another in the time of pyroptosis needs to be moreover researched.

Pyroptosis has not been entirely studied until now, albeit inevitable similarities to apoptosis and certain overlaps in machines have already been detected. As IPF develops, numerous modes of cellula demise 
may coexist and mutual effect on one another [29]. For instance, in our pattern, four genes (CASP8, CASP3, PYCARD, and NLRP3) are likewise decisive regulators in apoptotic pathways. Mostly, apoptosis highlights a full cellula plasma cytomembrane and is not free of contents, and doesn't straight induce phlogistic reactions, but pyroptosis reveals the reverse features [30]. We analyzed the differentially expressed genes inter different threat sets and detected that the DEGs were primarily relevant in the modification of morphology or physiology of other organisms.

CIBERSORT [31] is a ubiquitous method to calculate cellular immune infiltration. It uses the principle of linear support vector regression to DWFS the expression matrix of immune cell subtypes to estimate the abundance of immune cells. Whereas our analysis showed that the consequences of the two cohorts were not consistent, suggesting that the level of immune infiltration maybe not be an essential factor in the excellent prognosis of IPF sicks.

There are few recent studies on pyroptosis, peculiarly on its mechanics in IPF. Our research discerned three genes within the gasdermin clan that should be the performers of pyroptosis in IPF and three that can possess pyroptosis. We preliminary researched the prognostic importance of these PPGs and proved academic facilitate for to-be study. Nevertheless, as a consequence of a shortage of data, we are unable to validate if these calibrators (reported before) likewise play supplementary actors in pyroptosis passages in IPF, and this issue is worthy of complete researches moreover.

In short, our research proved which pyroptosis is cheek by jowl linked to IPF since the majority of the PPGs inter normal and IPF were dissimilarly expressed. Besides, the score produced from our threat prediction model ground on six PPGs was an alone threat element for forecasting IPF in the GSE28042 and the GSE70866-GPL17077 cohorts. The differentially expressed genes inter the shallow-threat and high-threat sets were linked with the modification of morphology or physiology of other organisms. Our research offers a new gene signature for forecasting the prognosis of IPF sicks and supplies a remarkable base for prospective researches of the correlations inter PPGs and signaling pathways in IPF, as well as immunity.

\section{Methods And Materials}

\section{Data sets}

We gained the RNA sequencing data of 75 IPF sicks and 19 controls and the homologous clinical traits from the GEO database (ID: GSE28042). The external validation cohort's RNA sequencing data and clinical features were downloaded from the GEO database (ID: GSE70866-GPL17077).

\section{Recognition of distinguishingly expressed PPGs (DEPPGs)}

We obtained 33 PPGs from an underlying study [32], and they are presented in Table S1. After downloading the expression matrix, the clone with the minimum deletion rate was selected as the expression value of uni-gene annotated. The "limma" package was employed to recognize DEPPGs with a pvalue $<0.05$. The DEPPGs are marked as follows: * if pvalue $<0.05$, $* *$ if pvalue $<0.01$, and $* \star *$ if pvalue 
$<0.001$. The protein-protein interaction network of DEPPGs was constructed by a searching tool for retrieval of interacting genes (STRING).

\section{Exploitation and verification of the PPGs prognostic pattern}

To evaluate the prognostic importance of the PPGs, we moreover hireling Cox regression analysis to assess the pertinence inter every gene and living conditions in the GSE28042 dataset. We set 0.01 as the cutoff point $p$-value to block false positives, and six survival-participant genes were ascertained for moreover analysis. The LASSO Cox regression pattern (R package "glmnet") was then used for straightening down the examinee genes and exploiting the prognostic pattern. Eventually, the six genes and their parameters were withheld, and the shallowest criteria settled the penalty parameter $(\lambda)$. The threat-score was computed after standardization (putting into use the "scale" function in R) of the GSE28042 expression profile, and the threat-score criterion was as follows: threat-Score $=\sum 6 \mathrm{iXix} \times \mathrm{Yi}(\mathrm{X}$ : parameters, Y: expression levels of a gene). The GSE28042 IPF sicks were uncoupled into shallow-threat and high-threat subgroups foundation on the mid-threat-score, and the overall survival time was contradistinguished inter the two subgroups via Kaplan-Meier analysis. The "dudi performed PCA ground on the 6-gene "signature.pca" and "inertia.dudi" function in the "ade4" R package. The "caret" R package was hireling to accomplish a ROC curve analysis. For the verification researches, an IPF cohort from the GSE70866-GPL17077 was hireling. The "scale" function likewise standardized the expression of each PPGs, and the threat-score was then figured out by the same equation used for the GSE28042 dataset. By putting into use the mid-threat-score from the GSE28042 dataset, the sicks in the GSE70866-GPL17077 dataset were likewise uncoupled into shallow-threat or high-threat subgroups, and these groups were whereafter contradistinguished to make sure the gene pattern.

\section{Independent prognosis studies of the threat-score}

We withdrew patients' clinical traits (gender, age, and status) in the GSE28042 and GSE70866-GPL17077 datasets. These variates were analyzed in association with the threat-score in our regression pattern. Single-variable and polynary Cox regression models were hirelings for the analysis.

\section{Functional enrichment studies and immune infiltration studies of the differentially expressed genes inter the shallow-threat and high-threat sets}

IPF sicks in the GSE28042 dataset were layered into two subgroups foundation the mid-threat-score. The differentially expressed genes inter the shallow-threat and high-threat groups were strained foundation precise criteria (padj $<0.05 \&|\log 2 \mathrm{FC}| \geq 1$ ). These differentially expressed genes, gene ontology, and Kyoto Encyclopedia of Genes and Genomes analyses were implemented by putting into use the "clusterProfiler" R software. The "Cibersort.R" package and "LM22.txt" were utilized to calculate the proportion of infiltrating immune cells.

\section{Statistical analysis}


One-factor analysis of square-deviation lent itself to contra distinguish the expression levels of genes inter the controls and IPFs. To arrive at the overall survival of sicks inter subgroups, we hired the KaplanMeier approach with a bilateral log-rank test. To appraise the autocephalous prognostic importance of the threat pattern, we did with single-variable and polynary Cox regression models. The Wilcoxon ranksum test compares the immune cellula infiltration inter the two groups. All statistic analyses were completed with R software (v4.1.1). The entire technological process graph is exhibited in Figure 10.

\section{Declarations}

\section{Ethics approval and consent to participate}

Not applicable

\section{Consent for publication}

Not applicable

\section{Availability of data and materials}

The datasets analysed during the current study are available in the GEO repository, https://www.ncbi.nlm.nih.gov/geo/query/acc.cgi?acc=GSE28042;

https://www.ncbi.nlm.nih.gov/geo/query/acc.cgi?acc=GSE70866.

The code to be utilized during the nowaday study are acquirable from the homologous author on the legitimate requirement.

\section{Competing interests}

All authors declare that they have no competitive benefits.

\section{Funding}

Not applicable

\section{Author Contributions}

Qiu-Di Zhang contributed to the conception, design, revision, and supervision of the study. Qian $\mathrm{He}$ contributed to drafting the manuscript and construction of prognostic patterns and data analysis. Yi Cao, Yue Zhao, Yi Shen, and Xiong Xu contributed to verifying prognostic patterns and data acquisition.

\section{Acknowledgments}

We want to acknowledge the GEO (https://www.ncbi.nlm.nih.gov/geo/) network for furnishing data.

\section{References}


1. Raghu G, Collard H R, Egan J J, et al. Fibrosis AEJACoIP (2011) An official ATS[R]. ERS/JRS/ALAT statement: idiopathic pulmonary fibrosis: evidence-based guidelines for diagnosis and management. Am J Respir Crit Care Med 183 (6): 788-824. https://doi. org/10.1164/rccm. 2009-040GL.

2. O’Dwyer D N, Ashley S L, Gurczynski S J, et al. Lung microbiota contribute to pulmonary inflammation and disease progression in pulmonary fibrosis[J]. American journal of respiratory and critical care medicine, 2019, 199(9): 1127-1138.

3. Hutchinson J, Fogarty A, Hubbard R, et al. Global incidence and mortality of idiopathic pulmonary fibrosis: a systematic review[J]. European Respiratory Journal, 2015, 46(3): 795-806.

4. Schaefer S C, Funke-Chambour M, Berezowska S. Idiopathic pulmonary fibrosis-epidemiology, causes, and clinical course[J]. Der Pathologe, 2020, 41(1): 46-51.

5. Behr J, Nathan S D, Wuyts W A, et al. efficacy and safety of sildenafil added to pirfenidone in patients with advanced idiopathic pulmonary fibrosis and risk of pulmonary hypertension: a double-blind, randomised, placebo-controlled, phase 2b trial[J]. The Lancet Respiratory Medicine, 2021, 9(1): 8595.

6. Kovacs S, Miao E. Gasdermins: effectors of pyroptosis. Trends Cell Biol. 2017;27:673-684. doi: 10.1016/j.tcb.2017.05.005.

7. Miao E, Rajan J, Aderem A. Caspase-1-induced pyroptotic cell death. Immunol. Rev. 2011;243:206214. doi: 10.1111/j.1600-065X.2011.01044.x.

8. Broz P, Pelegrín P, Shao F. The gasdermins, a protein family executing cell death and inflammation. Nat. Rev. Immunol. 2020;20:143-157. doi: 10.1038/s41577-019-0228-2.

9. Ding J, et al. Pore-forming activity and structural autoinhibition of the gasdermin family. Nature. 2016;535:111-116. doi: 10.1038/nature18590.

10. Feng S, Fox D, Man S. Mechanisms of gasdermin family members in inflammasome signaling and cell death. J. Mol. Biol. 2018;430:3068-3080. doi: 10.1016/j.jmb.2018.07.002.

11. Zhang Y, Chen X, Gueydan C, Han J. Plasma membrane changes during programmed cell deaths. Cell Res. 2018;28:9-21. doi: 10.1038/cr.2017.133.

12. Frank D, Vince J. Pyroptosis versus necroptosis: similarities, differences, and crosstalk. Cell Death Differ. 2019;26:99-114. doi: 10.1038/s41418-018-0212-6.

13. Liang $Q$, Cai $W, Z$ hao $Y$, et al. Lycorine ameliorates bleomycin-induced pulmonary fibrosis via inhibiting NLRP3 inflammasome activation and pyroptosis [J]. Pharmacological research, 2020, 158:104884.

14. Tang R, et al. Ferroptosis, necroptosis, and pyroptosis in anticancer immunity. J. Hematol. Oncol. 2020;13:110. doi: 10.1186/s13045-020-00946-7.

15. Liang Q , Cai W, Zhao Y, et al. Lycorine ameliorates bleomycin-induced pulmonary fibrosis via inhibiting NLRP3 inflammasome activation and pyroptosis [J]. Pharmacological research, 2020, 158:104884. 
16. Shi P, et al. Loss of conserved Gsdma3 self-regulation causes autophagy and cell death. Biochem. J. 2015;468:325-336. doi: 10.1042/BJ20150204.

17. Delmaghani $S$, et al. Hypervulnerability to sound exposure through impaired adaptive proliferation of peroxisomes. Cell. 2015;163:894-906. doi: 10.1016/j.cell.2015.10.023.

18. Shi J, Zhao Y, Wang K, et al. Cleavage of GSDMD by phlogistic caspases determines pyroptotic cell death[J]. Nature, 2015, 526(7575): 660-665.

19. Srinivasula S M. Ahmad M, Fernandes-Alnemri T, Litwack G, and Alnemri ES[J]. Molecular ordering of the Fas-apoptotic pathway: the Fas/APO-1 protease Mch5 is a CrmA-inhibitable protease that activates multiple Ced-3/ICE-like cysteine proteases. Proc Natl Acad Sci USA, 1996, 93: 14486-14491.

20. Krebs C H. J. 1999[J]. Ecological methodology, 2.

21. Blasche S, Mörtl M, Steuber H, et al. The E. coli effector protein NleF is a caspase inhibitor[J]. PloS one, 2013, 8(3): e58937.

22. Jiang M, Qi L, Li L, Li Y. The caspase-3/GSDME signal pathway as a switch between apoptosis and pyroptosis in cancer. Cell Death Discov. 2020;6:112. doi: 10.1038/s41420-020-00349-0.

23. Wang $Y$, et al. Chemotherapy drugs induce pyroptosis through caspase-3 cleavage of a gasdermin. Nature. 2017;547:99-103. doi: 10.1038/nature22393.

24. Nakanishi H, Kawashima Y, Kurima K, et al. NLRP3 mutation and cochlear autoinflammation cause syndromic and nonsyndromic hearing loss DFNA34 responsive to anakinra therapy[J]. Proceedings of the National Academy of Sciences, 2017, 114(37): E7766-E7775.

25. Nakanishi H, Kawashima Y, Kurima K, et al. NLRP3 mutation and cochlear autoinflammation cause syndromic and nonsyndromic hearing loss DFNA34 responsive to anakinra therapy[J]. Proceedings of the National Academy of Sciences, 2017, 114(37): E7766-E7775.

26. Lu B, Nakamura T, Inouye $K$, et al. Novel role of PKR in inflammasome activation and HMGB1 release[J]. Nature, 2012, 488(7413): 670-674.

27. Mitoma H, Hanabuchi S, Kim T, et al. The DHX33 RNA helicase senses cytosolic RNA and activates the NLRP3 inflammasome[J]. Immunity, 2013, 39(1): 123-135.

28. Wang $Y$, Ning $X$, Gao $P$, et al. Inflammasome activation triggers caspase-1-mediated cleavage of cGAS to regulate responses to DNA virus infection[J]. Immunity, 2017, 46(3): 393-404.

29. Fritsch $M$, et al. Caspase-8 is the molecular switch for apoptosis, necroptosis and pyroptosis. Nature. 2019;575:683-687. doi: 10.1038/s41586-019-1770-6.

30. Khan, I., Yousif, A., Chesnokov, M., Hong, L. \& Chefetz, I. A decade of cell death studies: breathing new life into necroptosis. Pharmacol. Therapeut. 107717 (2020).

31. Newman AM, Liu CL, Green MR, Gentles AJ, Feng W, Xu Y, et al. Robust Enumeration of Cell Subsets From Tissue Expression Profiles. Nat Methods (2015) 12(5):453-7. doi: 10.1038/nmeth.3337.

32. Ye Y, Dai Q, Qi H. A novel defined pyroptosis-related gene signature for predicting the prognosis of ovarian cancer[J]. Cell Death Discovery, 2021, 7(1): 1-11. 
Figures
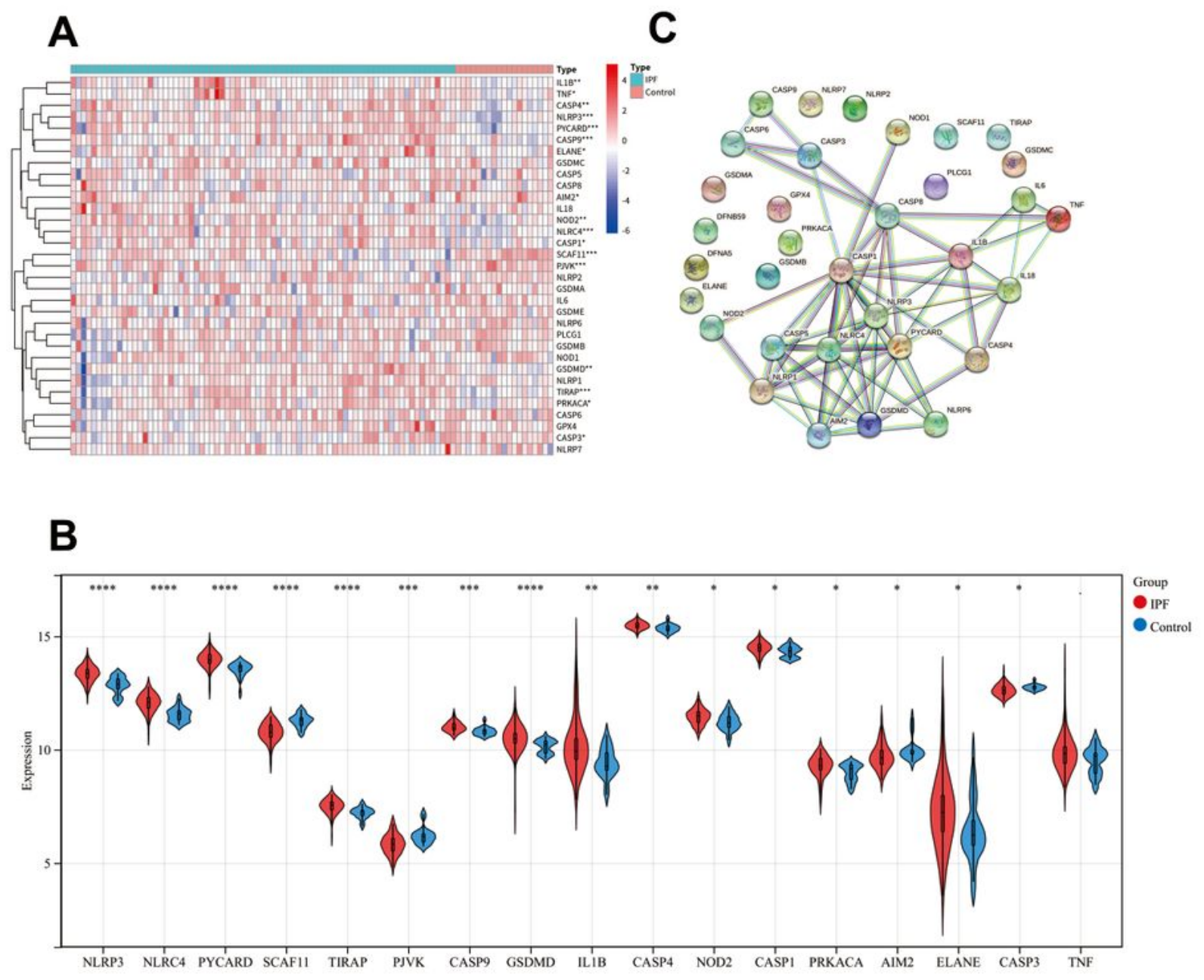

D

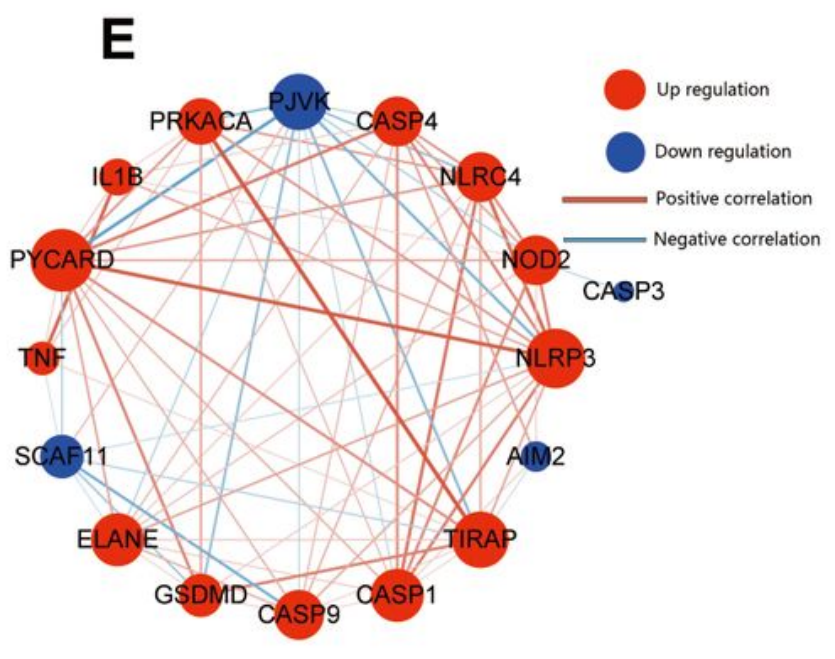

Figure 1

Expression levels of the 33 PPGs and the mutual effect in the middle of them. A Heat map (blue: shallow expression; red: high expression) of the PPGs inter the IPFs (blue) and the normals (red). Pvalues were shown as **pvalue $<0.01 ; * * *$ pvalue $<0.001$. B The expression of PPGs was significantly increased or 
decreased in 75 IPF contradistinguished with that in 25 normal tissues. C PPI network layout the mutual effect of the PPGs(interaction score = 0.9). D The intersection of hub PPGs and differentially expressed PPGs in the PPI network. E The association's network of the PPGs (red line: positive pertinence; blue line: negative pertinence. The thicker the line, the more significant the pertinence, and the more connected each node, the larger the node).
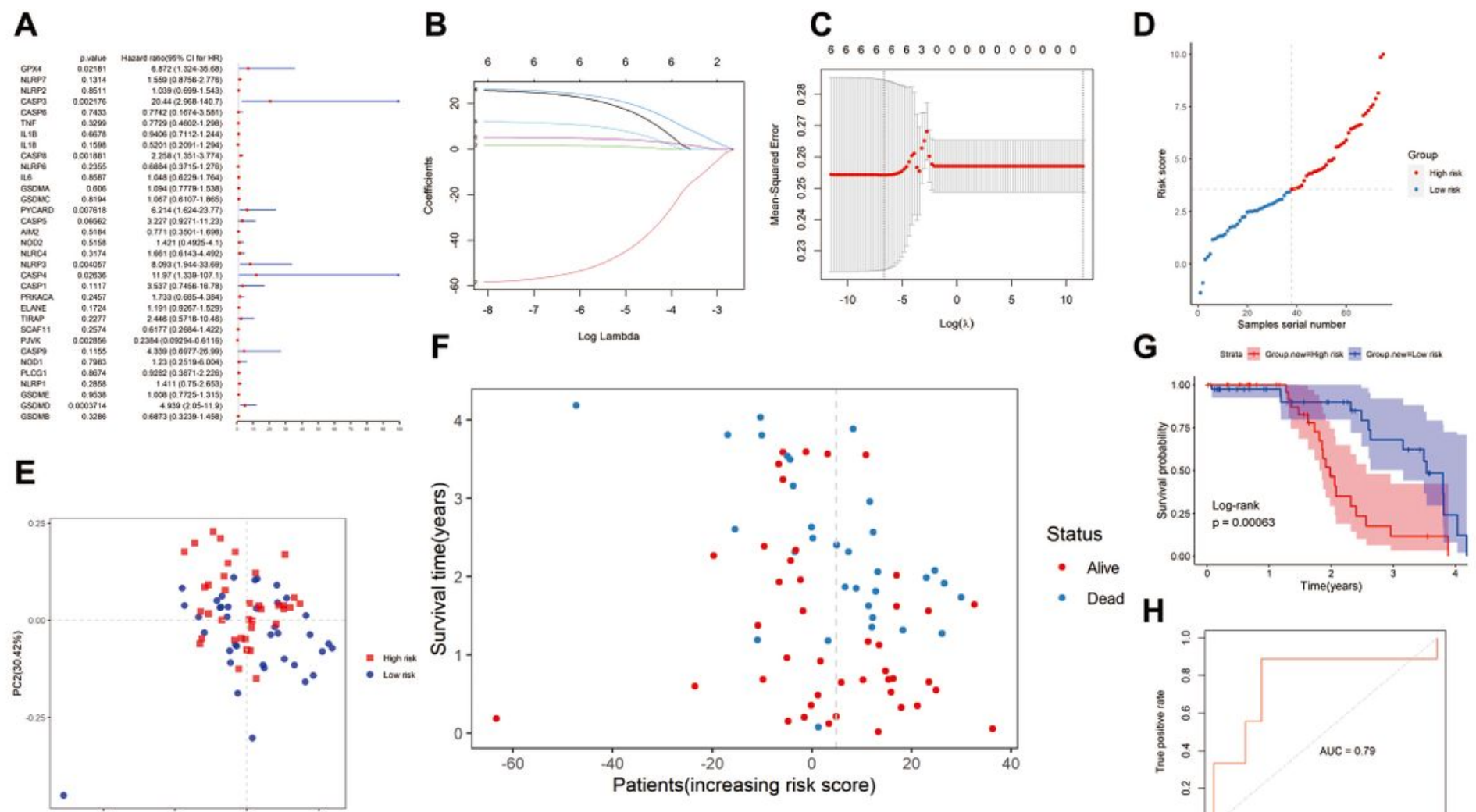

G
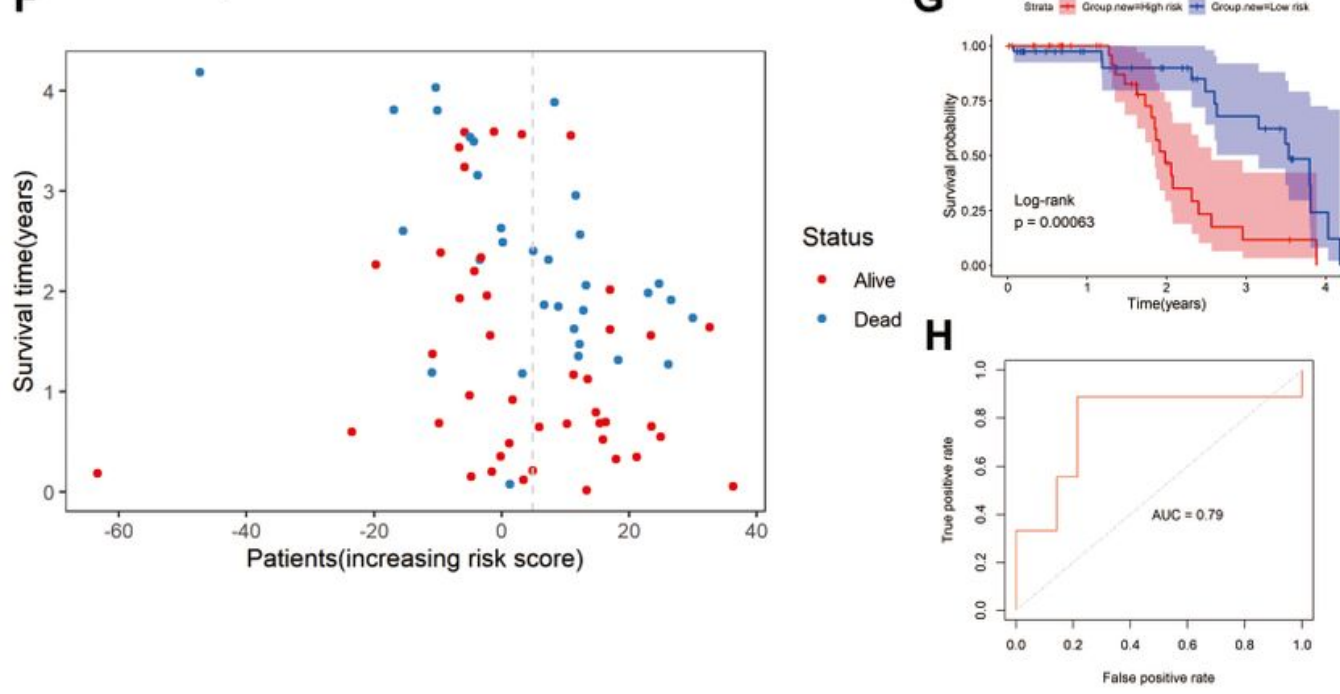

Figure 3

Establishment of a threat-score prediction model in the GSE28042 cohort. A Single-variable cox regression analysis of overall survival for each PPG and six genes with $P<0.01$. B LASSO regression of the 6 OS-participant genes. C Cross-validation for adjusting the argument pick in the least absolute shrinkage and selection operator regression. D Distribution of sicks ground on the threat-score. E PCA drawing for IPFs ground on the threat-score. F The living states for apiece sick (shallow-threat crowd: on the left aspect of the line of dashes; high-threat crowd: on the right aspect of the line of dashes). $\mathbf{G}$ Kaplan-Meier curves for the OS of sicks in the high-threat and shallow-threat sets. HROC curves proved the fatidic potency of the threat score. 

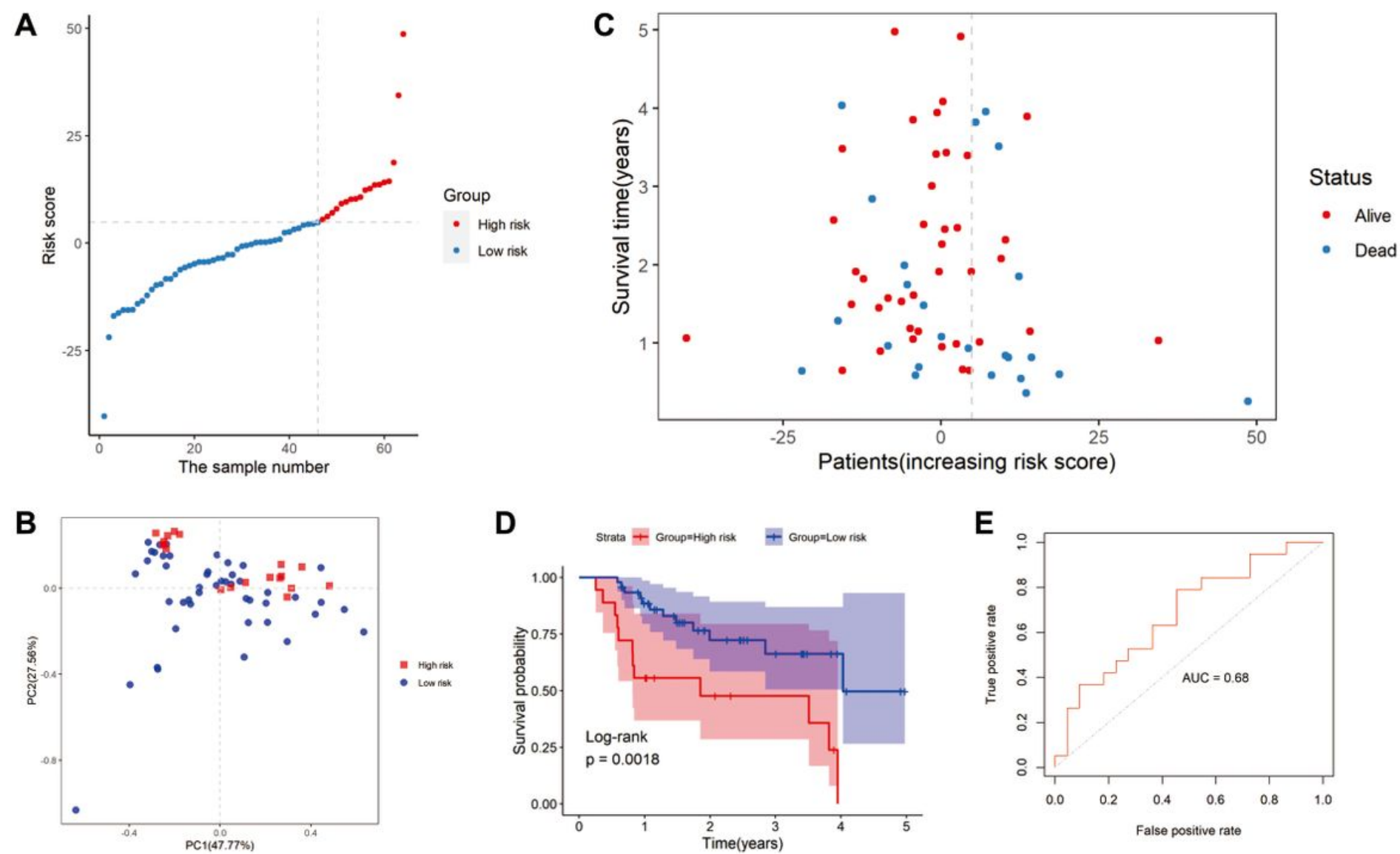

Figure 4

Verification of the threat pattern in the GSE70866-GPL17077 dataset. A Distribution of sicks in the GSE70866-GPL17077 dataset ground on the mid-threat-score in the GSE28042 dataset. B PCA was drawing for IPFs. C The living states for the respective sick (shallow-threat crowd: on the left aspect of the line of dashes; high-threat crowd: on the right aspect of the line of dashes). D Kaplan-Meier curves to compare the overall survival time inter shallow-threat and high-threat communities. E ROC curves for IPFs. 
A

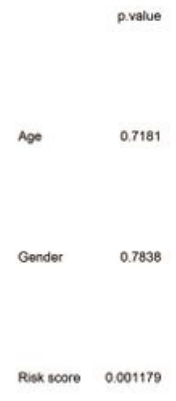

C

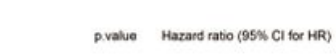

Age $\quad 0.87737 \quad 0.9963(0.9501-1.045)$

onoms

Risk score 0.00124

hazard ratio (95\% Cl for HR)
B

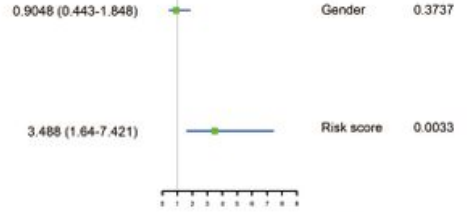

D

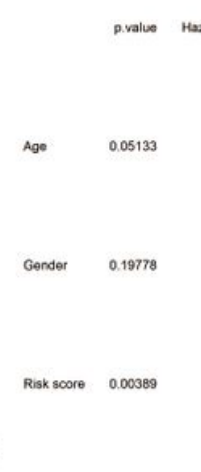

E

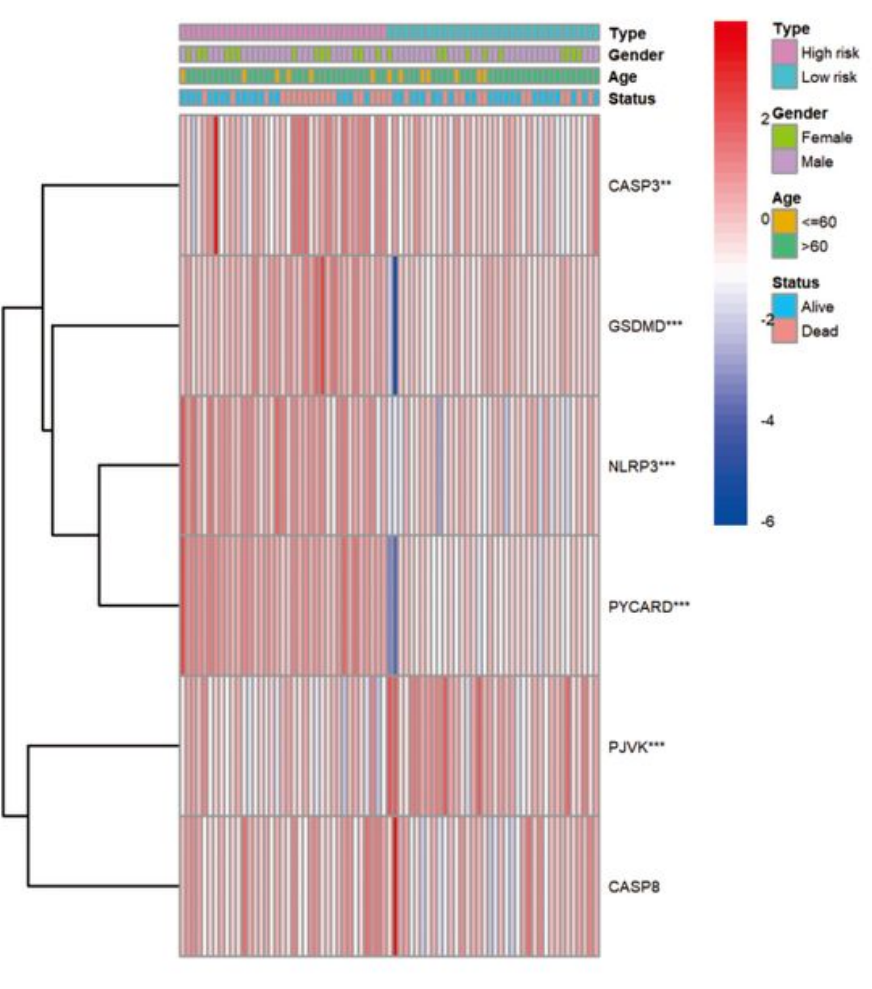

\section{Figure 5}

single-variable and polynary Cox regression analyses for the threat-score. Single-variable analysis for the GSE28042 dataset (gender: female, male). B polynary analysis for the GSE28042 dataset. C singlevariable analysis for the GSE70866-GPL17077 mate (gender: female, male). D polynary analysis for the GSE70866-GPL17077 dataset. E Heat map (blue: shallow expression; red: high expression) for the joints inter clinical traits and the threat groups. 


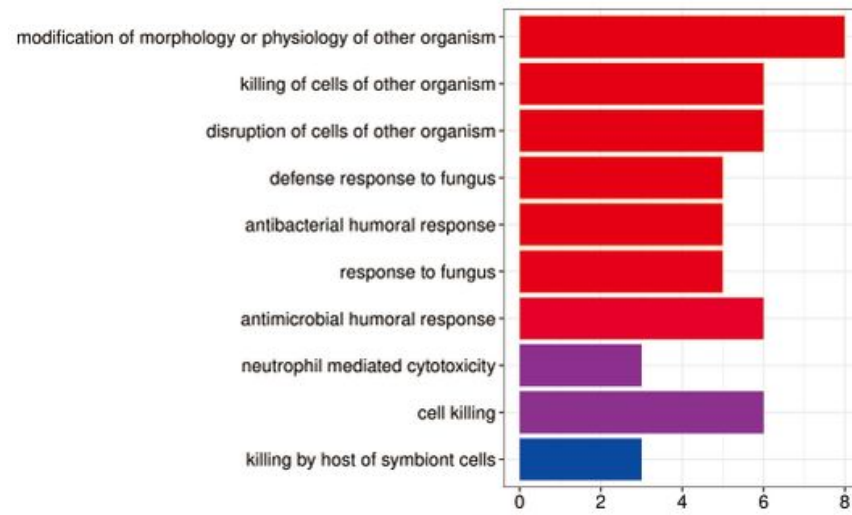

C
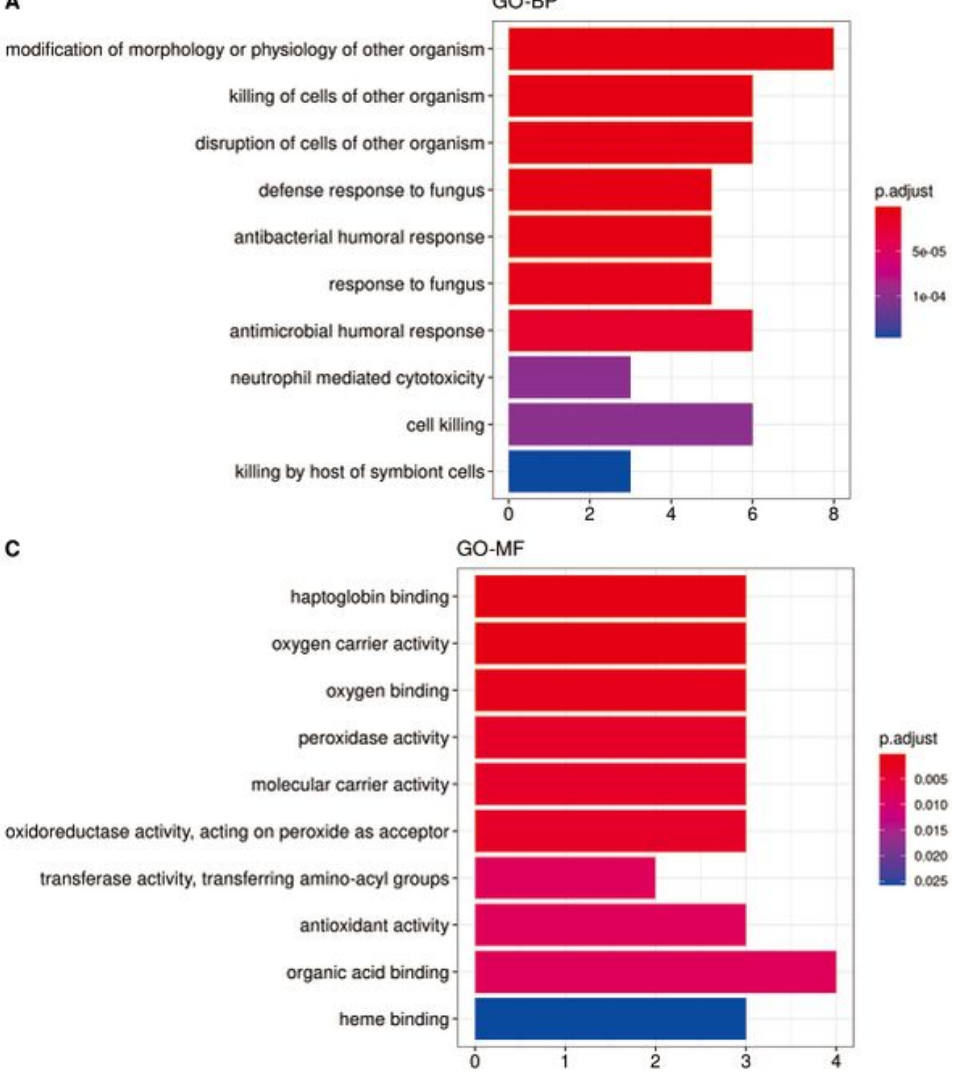

GO-CC

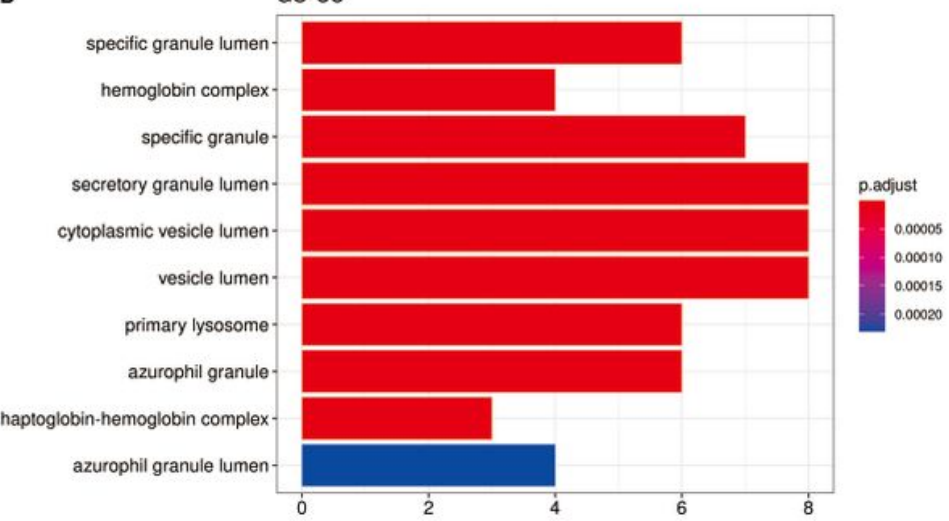

D

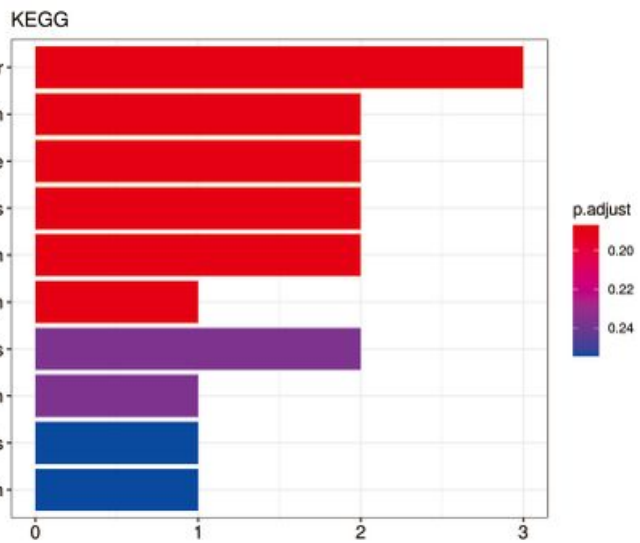

Figure 6

Functional enrichment analysis ground on the differentially expressed genes inter the two-threat sets in the GSE28042 cohort. A, B, C Barplot for GO enrichment (the more extended bar implies the more genes concentrated, and the adding deepness of red implies the deviations were additional evident). D Barplot for KEGG pathways (the more extended bar implies the more genes concentrated, and the adding deepness of red implies the deviations were additional evident).
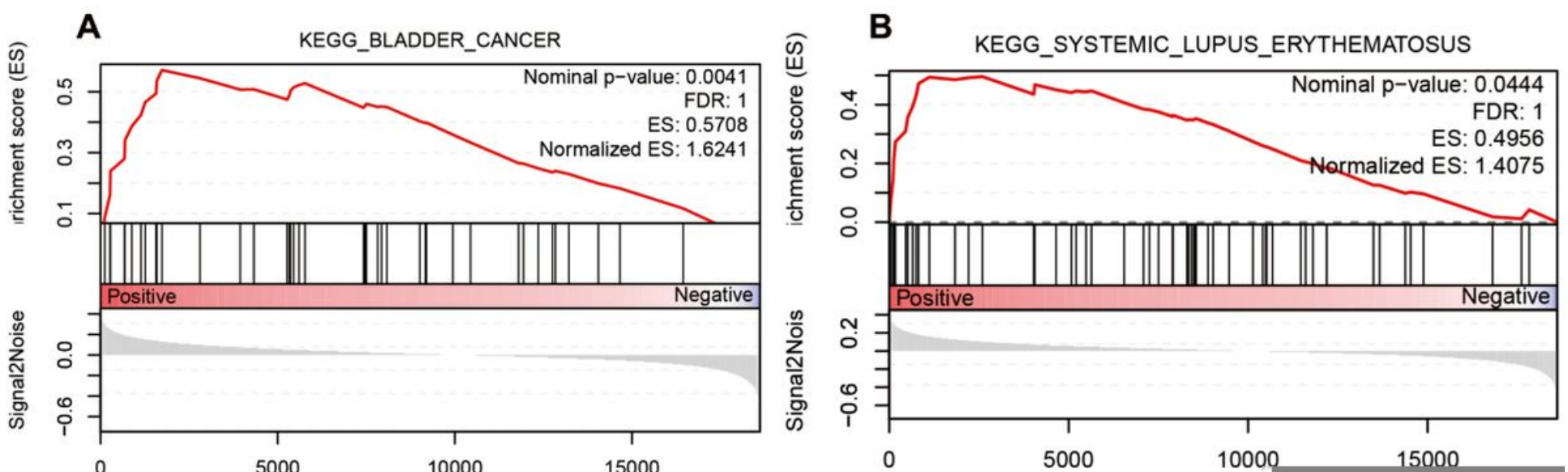

Figure 7 
GSEA showed the significantly enriched KEGG gene sets in the high-threat-scores ground on the GSE28042 database.
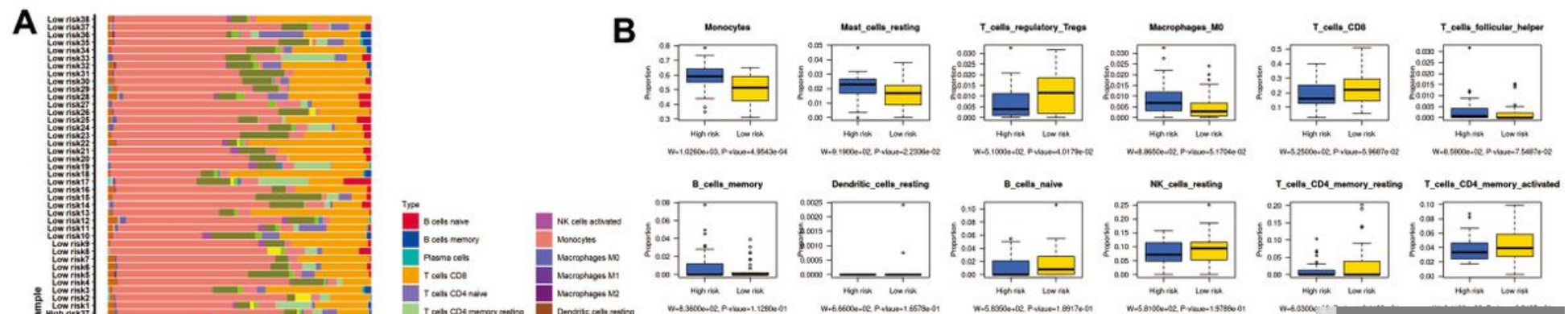

\section{Figure 8}

To compare the proportion for immune cells in the GSE28042 cohort. A Barplot of cellula proportion. B To compare the abundance scores of 22 types of immune cellular inter shallow-threat (orange box) and high-threat (grey box) groups.
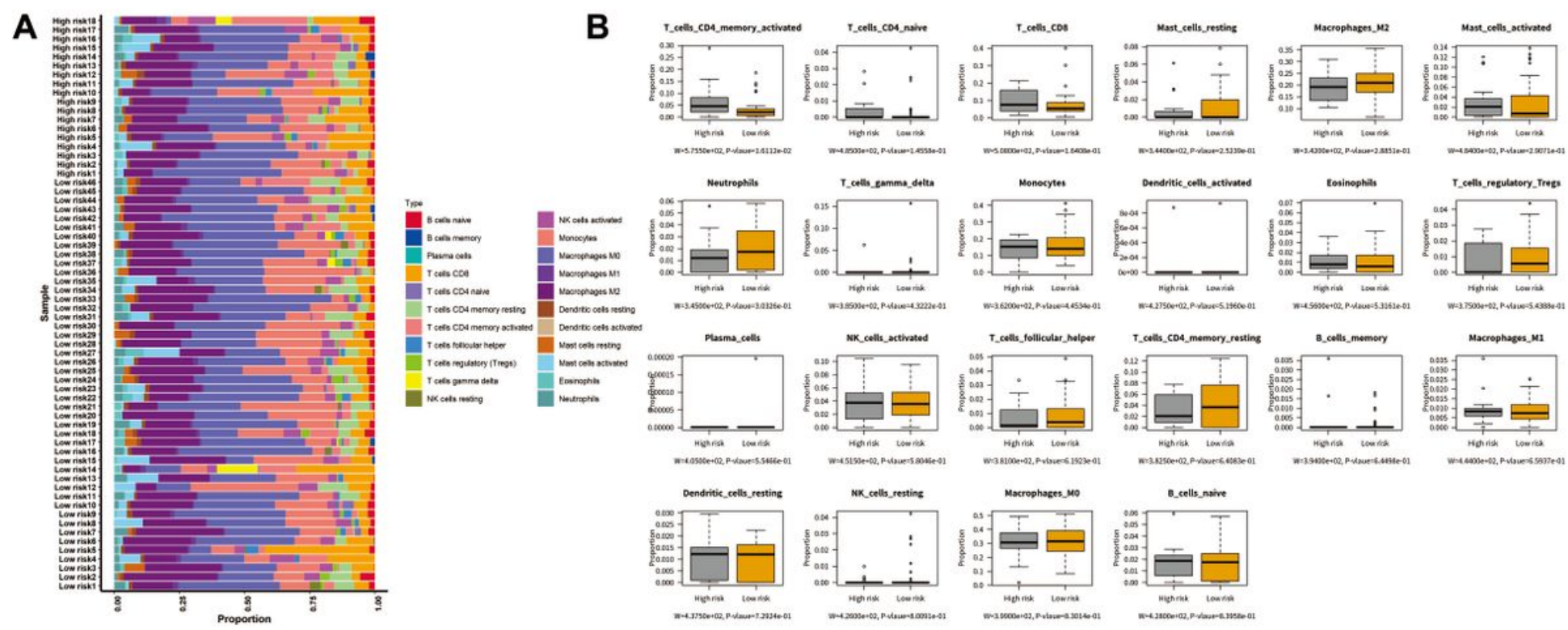

\section{Figure 9}

To compare the proportion for immune cells in the GSE70866-GPL17077 cohort. A Barplot of cellula proportion. B To compare the abundance scores of 22 descriptions of immune cellular inter shallow- 
threat (orange box) and high-threat (grey box) groups.

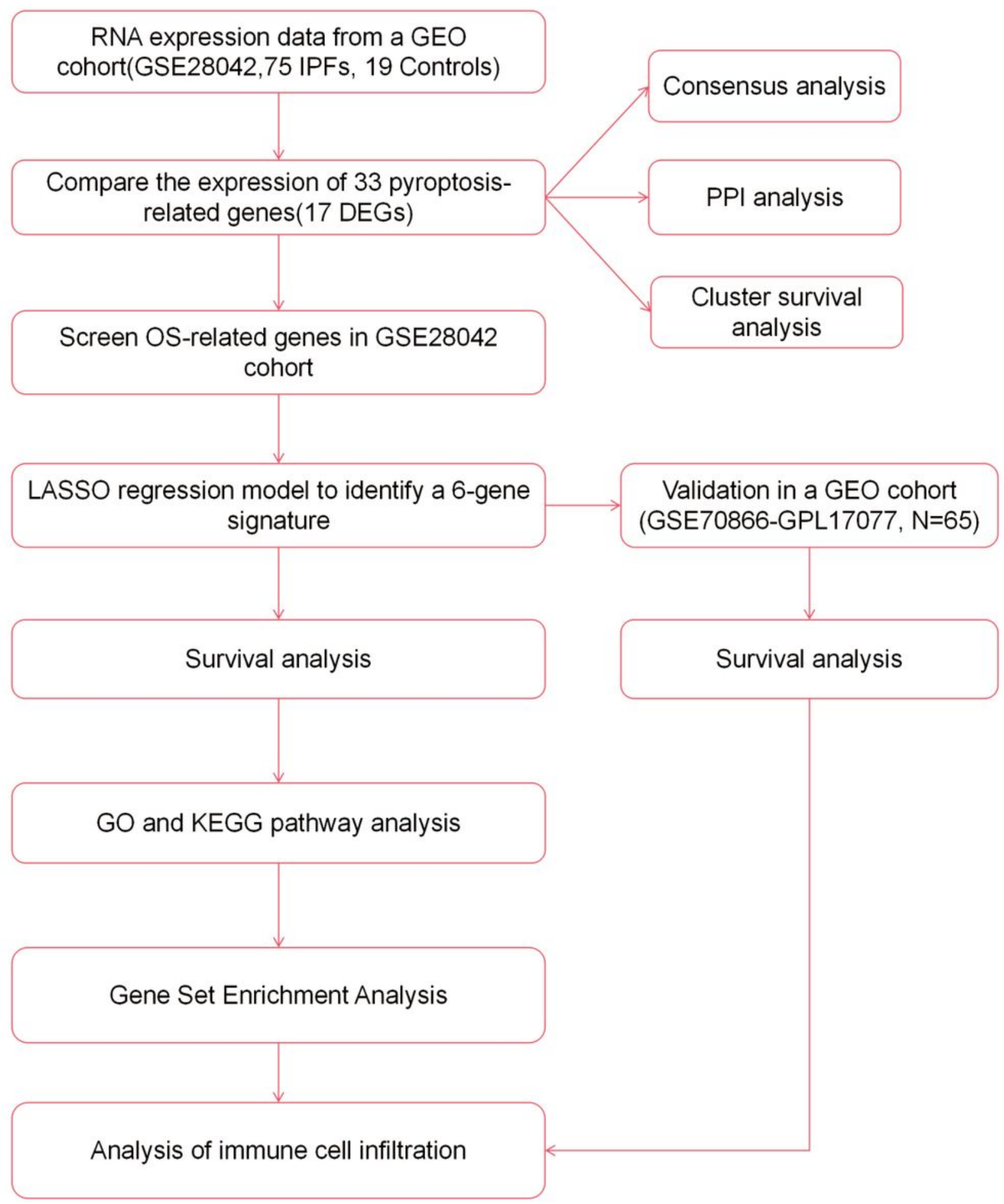

Figure 10

Technology roadmap. The precise work process of analysis of data.

\section{Supplementary Files}


This is a list of supplementary files associated with this preprint. Click to download.

- TableS1.xlsx

- TableS2.xIsx 